\title{
Evaluation of a Cystic Fibrosis Transition Program From Pediatric to Adult Care
}

\author{
Sarah R. Chaudhry, MPH, Meghan Keaton, MD, and Samya Z. Nasr, MD*
}

\begin{abstract}
Summary. Purpose: As the cystic fibrosis (CF) patient population median survival increase, the need for transitioning their care to adult care centers increase as well. We have a structured transition program since the early 1980s. The purpose of this study is to evaluate the experiences and opinions of patients in our adult CF center who went through a formal transition versus those who did not, in an attempt to evaluate the overall process and to identify means for improvement. Methods: A questionnaire was given to adult CF patients at the University of Michigan during a clinic visit or inpatient hospitalization, after consent was obtained. Inclusion criteria included diagnosis of CF in childhood, previous care in a pediatric center, and current participation in our adult CF clinic. Results: A total of 91 patients completed the questionnaire. 44 went through our structured transition program. On average, patients who participated in a formal transition process had higher satisfaction with both CF programs, perceived health status, and independence but no differences in their level of anxiety about transferring to the adult program. Patient opinions regarding when to transfer care were considered more often in patients who participated in a transition program ( $88 \%$ vs. $62 \%$ and $P=0.02)$. Conclusion: Although structured transitions programs do not appear to decrease patient anxiety during this stressful period, it does appear to improve patient satisfaction, perceived health status, and independence. These findings suggest that establishing a transition program could be important in optimizing patient health, continuity of care and improving adherence. Pediatr Pulmonol. 2013; 48:658-665. ๑ 2012 Wiley Periodicals, Inc.
\end{abstract}

Key words: transition; cystic fibrosis center; transfer of care.

Funding source: Cystic Fibrosis Foundation.

\section{INTRODUCTION}

As advancements in medical technology and health care are made, survival of children with chronic disorders continues to improve and mortality rates decrease. Currently, it is estimated that $90 \%$ of children with chronic disorders reach adulthood, highlighting the need to implement the transition of care from pediatric to adult specialists. ${ }^{1}$ The goal of a planned transition is to reduce interruption of care, maximize well-being, and improve quality of life. ${ }^{2}$ Unfortunately, this period of transition typically occurs when patients are already struggling with the biological, emotional, and psychological changes that accompany adolescence, which can be particularly more stressful for chronically ill patients. ${ }^{3}$ In addition, at the age of transition, patients may be facing issues regarding disruption of care and loss of health insurance as well as trying to develop self-sufficient disease management skills. For these reasons, a well-timed transition from pediatric to adult oriented health care is specific to each person and ideally occurs between the recommended ages of 18 and 21 years. $^{2}$

The American Academy of Pediatrics, American Academy of Family Physicians, and American College of Physicians recently published a clinical report supporting the health care transition from adolescence to adulthood. $^{2}$ It stated that the goal of a planned health care transition is to maximize lifelong functioning and well-being for all youth, including those who have special health care needs and those who do not. ${ }^{2}$ The transition process should be high-quality, developmentally appropriate, and it should provide uninterrupted care. Several studies addressing the transition process for patients with chronic diseases have been published. ${ }^{1-16}$

Division of Pediatric Pulmonology, C.S. Mott Children's Hospital, University of Michigan, Ann Arbor, Michigan 48309.

Conflict of interest: None.

*Correspondence to: Samya Z. Nasr, MD, Division of Pulmonology, Department of Pediatrics, University of Michigan Health System, 1500 E. Medical Center Dr., L2221 Women's, Ann Arbor, MI 48109-0212

E-mail: snasr@umich.edu

Received 24 January 2012; Revised 6 July 2012; Accepted 10 July 2012.

DOI 10.1002/ppul.22647

Published online 8 August 2012 in Wiley Online Library

(wileyonlinelibrary.com). 
And recently, a metasummary analysis of quantitative and qualitative studies of patients' perspective on factors facilitating transition from child-centered to adultcentered health care was conducted to summarize research that has been done in this area. The findings fell into four categories: 1-patients' feelings and concerns, 2-patients' recommendations about transition, 3-outcomes after transition, and 4-effects of the mode of transfer, which provided helpful insight into the primary factors that patients felt facilitated a successful transition to adult-centered care. ${ }^{4}$

However, despite significant efforts to develop smooth transition process for all patients with chronic diseases, individuals with disabilities report having significant problems with access, even though they are the largest group of health care users in the so-called vulnerable population. ${ }^{17}$ Common barriers to transitioning include patient or family reluctance, poor communication between pediatricians and adult practitioners, internists' discomfort with treating unfamiliar diseases and structural hospital problems. ${ }^{1,3}$ Unfortunately, patients that transfer care to an adult clinic via a poor or non-existent transition program are more likely to be lost to follow-up. These patients usually do not seek health care until a medical problem arises. ${ }^{7}$ In addition, poor transition programs have been found to have significant negative effects on morbidity and mortality in young adults. ${ }^{5,7}$ Recently, a systematic review of studies that evaluated health outcomes following transition programs was done. ${ }^{14}$ The study concluded that the most commonly used strategies in successful programs were patient education and specific transition clinics. ${ }^{14}$

One chronic disease that has really come to the forefront regarding the need for structured transition has been cystic fibrosis (CF). Decades ago, medical care for these patients was centered on a goal of ensuring survival to adulthood. However, given significant advancements in $\mathrm{CF}$ medical therapy, survival in this population has steadily increased with the current predicted median survival age of 38 years. ${ }^{15}$ In addition, based on national data, the Cystic Fibrosis Foundation (CFF) estimates that at least $47 \%$ of the total population of CF patients in the United States is now 18 years or older. ${ }^{15}$ To optimize care for this growing adult population, the CFF mandated in 1996 that CF centers develop a model to provide age-appropriate $\mathrm{CF}$ care to maintain accreditation, with the expectation that patients be transitioned to adult providers by 21 years of age. Several studies evaluating $\mathrm{CF}$ transition programs nationally and internationally have been published. ${ }^{3,8-13,16}$

The objective of this study is to evaluate the transition program at the University of Michigan CF Center through patients' perceptions. The goal is that, following the evaluation, further modification of the transition program will be conducted accordingly. The University of Michigan CF center has a long-standing transition program that was established in $19800^{3}$ This program was evaluated in 1992. Since then the transition process was changed to accommodate input obtained from that evaluation. The main change that occurred after the initial evaluation was to have the adult pulmonologist, not the fellow, come in to the pediatric pulmonary clinic to meet the patients for one to three visits followed by transitioning the patients to the adult clinic. In this study, in order to re-evaluate our patients' perceptions of the transition program, we used a modified version of the questionnaire that was utilized in our previous study. The questionnaire allowed patients to respond to questions about their pediatric care, their experience with the transition process, and the adult CF program. The patients were also asked for suggestions to improve the transition program. The hypotheses of the study were: 1 -patients that went through the transition program would be more satisfied and have fewer concerns about the adult program compared to patients that did not go through the transition program. 2-Patients who participated in the transition program would have a better perceived health status before and after the transition program than those who did not participate. 3-Patients who participated in the transition program would be more satisfied with their pediatric program.

\section{METHODS}

\section{Transition Process Description}

A. Preparing for transition

(1) The transition process is introduced to patients and families at time of diagnosis or at their first visit to our center.

(2) As patients get older, the team members gradually shift from interacting with parents to interacting with patients in clinic visits and during inpatient care.

(3) In the early teenage years, the team members focus on reeducation of the teens and families about the disease process and purpose of medications and treatment plan.

(4) Focus on promotion of self-management is led by our social workers and psychologists. Independent visits are offered and encouraged.

(5) Intervention with the teens and families to help improve adherence to treatment and resolve conflicts is done by social workers and psychologists during clinic visits and hospitalizations.

(6) The adult CF team is an integral part of our annual newsletter and family education function to demonstrate collaboration between the two programs to patients and families. 
(7) Social workers evaluate patients for transfer readiness through mid to late teenage years.

(8) In preparation for transition, the pediatric team re-introduces the transition process to patients when they reach their early teens, a time when they are gaining more independence.

(9) Starting at mid teenage years, patients are periodically discussed in the monthly meeting between the pediatric and adult pulmonologists and transition coordinators (social workers).

\section{B. Active transition process}

(1) The transition coordinator and the pediatric pulmonary team discuss the process with the patient and family. The adult pulmonologists are reviewed and a choice of an adult pulmonologist is made.

(2) A patient ready for transition is reviewed in the monthly pediatric-adult $\mathrm{CF}$ meeting. Medical records are reviewed by the adult pulmonologist prior to meeting the patient and family.

(3) This is followed by an appointment in the pediatric clinic attended by the pediatric and adult pulmonologist to provide opportunities for interaction between the medical team and patients and families. The adult pulmonologist meets the patient and family with the pediatric pulmonologist in the pediatric clinic.

(4) If the patient and family are not ready after this joint clinic session, the session is repeated until they are ready, for up to three visits.

(5) A tour of the adult inpatient and outpatient facilities is offered prior to the transition to adult care.

\section{Study Design}

Approval to conduct our study was granted by the University of Michigan Institutional Review Board. A questionnaire was developed by modifying the previous questionnaire that was used in the first study. ${ }^{3}$ The questionnaire was given to $\mathrm{CF}$ patients attending the adult CF Center at the University of Michigan Health System during a clinic visit or inpatient hospitalization to complete after consent was obtained. Inclusion criteria included diagnosis of $\mathrm{CF}$ in childhood, previous care in a pediatric center, and current participation in our adult CF Center. Patients were questioned regarding their pediatric and adult $\mathrm{CF}$ care experience and the transition process that they went through.

\section{Patient Population}

Patients attending the adult $\mathrm{CF}$ center were invited to participate in the study between January 2010 and December 2010. Sixty percent of the total adult CF center patients participated in the study. The patient population surveyed was transferred from pediatric to adult care an average of 8.4 years before the time of the study.

Patients were divided into two groups:

(1) Patients that went through a transition process:

A. From the Pediatric to the Adult Program at the University of Michigan CF Center. The formal transition process was applied.

B. From an outside Pediatric Program to $\mathrm{U}$ of $\mathrm{M}$ Adult Program with informal transition process. This informal transition process was not specified by the patients and patients came from multiple different $\mathrm{CF}$ pediatric centers.

(2) Patients that were transferred to our adult CF Program without any transition or orientation process to the adult program.

\section{Questionnaire}

The questionnaire was modified from the one that was used in the earlier study. ${ }^{3}$ The original questionnaire included questions about the University of Michigan pediatric and adult CF programs, the role of the social workers in the transition process, and the number of times patients met with the adult pulmonary fellow in the pediatric clinic. The modifications included questions about the pediatric programs patients attended and the transition process (if any) that the patient went through. In addition, patients were asked about anxiety, satisfaction with care, perceived health status, and success in achieving independence both before and after the transition process. In both the original and modified questionnaires, there were questions about patient satisfaction with the transition process and their feelings about the different aspects of the pediatric and adult programs. There were several open ended questions to help in obtaining patient feedback and suggestions for improving the transition process. The total number of questions was 20 in the modified questionnaire, with five questions added to address the issues listed above. Replies to the questionnaire were kept confidential.

\section{Statistical Analysis}

Data was analyzed using bivariate analysis to analyze the relationship between the key outcomes (anxiety, satisfaction with care, perceived health status, and achieving independence). All study patients were asked to rate each of the four outcomes before and after 
transfer of care to the adult center on a scale from 1 to 5 (high/excellent $=5$ and low/poor $=1)$. Key outcomes consisted of single measures (one-item questions were scored on a five-point Likert scale). Cronbach's alpha was computed as a descriptive tool to measure how interconnected the four outcomes were. It can also measure the internal consistency and reliability of the outcome measures, if they were combined. The Cronbach's alpha values were 0.54 and 0.44 for "before" and "after" measures, respectively, which could indicate lack of internal consistency. However, since a combination of the four outcome measures was not done in this analysis, Cronbach's alpha was only used as a descriptive tool. While bivariate analyses can describe the relationship between a predictor (e.g., participation in transition program) and a response (e.g., anxiety after program), confounding variables may distort the nature of the relationship. In order to address issues of possible confounding, we use multiple regression models to control for gender and severity. The threshold of statistical significance was set at $P=0.05$.

\section{RESULTS}

\section{Study Population}

A total of 91 patients completed the questionnaire. Forty-four out of 91 study patients went through a structured transition program when transferring care from a pediatric to adult $\mathrm{CF}$ center and are referred to as "Participants." Out of the 44 transition program participants, 10 went through a transition program at an institution other than the University of Michigan. Forty-seven out of 91 study patients did not go through a structured transition program and are referred to as "Non-Participants." Table 1 summarizes the patient demographics for both the participant and non-participant groups.

\section{Comparing Participants to Non-Participants}

All study patients were asked if they felt that their opinion was considered regarding when they should

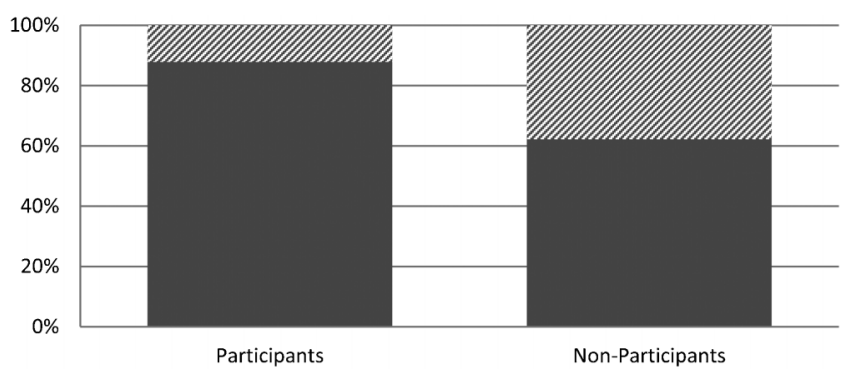

Fig. 1. Consideration of patient opinion on when to transfer care. \%/, opinion not considered; $\square$, opinion considered.

transfer to the adult CF clinic. Figure 1 illustrates their responses. Patients who participated in the program had greater odds of having their opinion considered $(\mathrm{OR}=4.30, \quad P=0.02)$. Patient opinions regarding when to transfer care were considered more often in patients who participated in a transition program $(88 \%$ vs. $62 \%$ and $P=0.02$ ). Patients who proceeded through a formal transition program reported a higher level of satisfaction with care before transferring to adult care $(P=0.01)$. Meanwhile, these patients did not show significantly more satisfaction with the adult program $(P=0.12)$. That indicates that patients are more satisfied with their pediatric center if the center has a structured transition process. Patients who participated in a structured transition process reported significantly higher perceived health status both before and after transition ( $P=0.01$ and 0.04 , respectively). Also, satisfaction with the preparation for the transition process and pediatric center were higher in the group of patients that went through the transition program.

A bivariate analysis was conducted to describe the relationship between key outcomes of interest (anxiety, satisfaction with care, perceived health status, and achieving independence) and participation in the transition program. Table 2 shows the bivariate relationships between each outcome and participation in the transition program. This analysis was done to test whether there is a significant difference between the two groups.

TABLE 1-Patient Demographics

\begin{tabular}{lccc}
\hline & All study patients & Transition program participants & Transition program non-participants \\
\hline Females & 41 & 20 & 21 \\
Males & 50 & 24 & 26 \\
Age (years) & $30.8 \pm 9.3$ & $27.6 \pm 7.2$ & $33.9 \pm 10.1$ \\
Age at CF diagnosis & $2.5 \pm 4.0$ & $2.8 \pm 4.0$ & $2.1 \pm 4.1$ \\
Age at transfer of care & $20.4 \pm 4.6$ & $19.1 \pm 2.1$ & $21.6 \pm 6.0$ \\
BMI (range) & $22.3(14.9,35.0)$ & $22.7(16.1,35.0)$ & $22.0(14.9,31.9)$ \\
$\mathrm{FEV}_{1} \%$ (range) & $61.0(15.0,111.0)$ & $66.8(15.0,111.0)$ & $56.0(15.0,105.0)$ \\
$\mathrm{FEF}_{25-75}$ (range) & $45.9(4.0,197.0)$ & $53.3(4.0,156.0)$ & $39.0(6.0,197.0)$ \\
\hline
\end{tabular}


TABLE 2-Relationship Between Key Outcomes and Participation in Transition Program

\begin{tabular}{lccc}
\hline Outcome & $\begin{array}{c}\text { Mean for } \\
\text { participants }\end{array}$ & $\begin{array}{c}\text { Mean for } \\
\text { non-participants }\end{array}$ & $P$-value \\
\hline Anxiety before & 2.50 & 2.49 & 0.97 \\
Anxiety after & 1.98 & 2.04 & 0.80 \\
Satisfaction before & 4.59 & 3.96 & 0.01 \\
Satisfaction after & 4.57 & 4.20 & 0.12 \\
Perceived health status before & 4.05 & 3.45 & 0.01 \\
Perceived health status after & 3.97 & 3.51 & 0.04 \\
Independence before & 4.35 & 4.20 & 0.44 \\
Independence after & 4.52 & 4.33 & 0.26 \\
\hline
\end{tabular}

On average, patients participating in the program had higher satisfaction, perceived health status, and independence. These averages were significantly different for the three groups: satisfaction before, satisfaction after, and perceived health status before.

There may be imbalances in the data on other variables which could alter the estimated effect of participating in a transition program. For example, when examining the relationship between participation and severity of $\mathrm{CF}$, we observed that patients with more severe disease were participating in a transition program less often than those with less severe disease. To address these issues, we included other predictors (gender, severity) in a multiple regression model. The multiple regression models tell virtually the same story as the bivariate regression models (Table 3). Participation is associated with an increase in satisfaction and an increase in perceived health status. There was no strong relationship between participation and either independence or anxiety.

All patients were asked questions regarding the care they received at their pediatric clinics. Patients were asked if they talked to someone in their pediatric clinic about leaving the clinic, leaving their physician, going to a new clinic and meeting a new doctor. Table 4 com-

TABLE 3-Multiple Regression Analysis Comparing Participants to Non-Participants

\begin{tabular}{lrrrrr}
\hline & \multicolumn{5}{c}{ Std. } \\
Outcome & Estimate & error & $t$-value & $P$-value & $R$-squared \\
\hline Anxiety before & 0.11 & 0.33 & 0.34 & 0.737 & 0.04 \\
Anxiety after & -0.01 & 0.27 & -0.05 & 0.958 & 0.03 \\
Satisfaction before & 0.65 & 0.25 & 2.59 & 0.011 & 0.12 \\
Satisfaction after & 0.43 & 0.24 & 1.80 & 0.075 & 0.08 \\
$\begin{array}{l}\text { Perceived health status } \\
\quad\end{array}$ & 0.48 & 0.23 & 2.07 & 0.041 & 0.12 \\
$\quad$ before & 0.30 & 0.22 & 1.38 & 0.170 & 0.15 \\
$\quad \begin{array}{l}\text { Perceived health status } \\
\text { after }\end{array}$ & 0.09 & 0.20 & 0.46 & 0.645 & 0.04 \\
$\begin{array}{l}\text { Independence before } \\
\text { Independence after }\end{array}$ & 0.18 & 0.18 & 0.99 & 0.326 & 0.02 \\
\hline
\end{tabular}

pares responses to these questions between participant and non-participant groups. For each question, a Fisher's test was performed to determine if there is a significant relationship between participation and the question of interest.

\section{Evaluation of the $\mathbf{U}$ of $\mathbf{M}$ Transition Program}

Out of the 44 transition program participants, 10 went through a transition program at different institutions other than the University of Michigan. The responses of these 10 study patients were not included in the evaluation of the $\mathrm{U}$ of $\mathrm{M}$ Transition Program. Figure 2 indicates study patient responses in terms of usefulness for five different experiences that participants may have encountered throughout the transition program.

\section{DISCUSSION}

For patients with disabilities and their families, there are three critical points in their lives: time of diagnosis, puberty, and the transition from adolescence to adulthood. ${ }^{1}$ Each of these critical points has to be handled with great care. ${ }^{1}$ As increasing numbers of young people with chronic illness is reaching adulthood, their medical care should be transitioned to adult providers. ${ }^{5}$ Several articles evaluated the transition process for patients with chronic diseases. ${ }^{4-7,14}$ A metasummary study indicated that a successful transition process should be presented as a normative event. It should be clarified and discussed to meet patient expectations and preparation should be started early. In addition, there should be joint planning with patients in order to provide the patient with an optimal transition environment and resources. ${ }^{4}$ A systematic review of several transition studies concluded that the most commonly used strategies in successful transition processes were patient education and specific transition clinics. ${ }^{14}$ With improvement in CF care almost half the patient population is reaching adulthood. ${ }^{15}$ It is crucial for people with $\mathrm{CF}$ to transition from pediatric to adult care. The goal of a well-planned transition program is to maximize lifelong functioning and well-being for all patients. ${ }^{2}$ In addition, a well-planned transition program might reduce interruption of care.

Even though the CFF strongly recommends that each $\mathrm{CF}$ Center should have a clear plan for transitioning their patients to adult care, ${ }^{15}$ the transition process has not been standardized between centers. ${ }^{8,11}$ That is true for other chronic diseases as well. Wide spread implementation of health transition supports as a basic standard of high-quality care has not been realized. Only limited progress has been documented to date. ${ }^{2}$

A structured transition program should include coordination and preparation between the pediatric $\mathrm{CF}$ 
TABLE 4-Pediatric Clinic Comparison Between Participant and Non-Participant Groups

\begin{tabular}{lccr}
\hline Patient talked to someone in clinic about. . & Participants in transition program (\%) & Non-Participants in transition program $(\%)$ & $P$-value \\
\hline Leaving the pediatric clinic & 75.0 & 37.8 & 0.001 \\
Leaving their pediatric physician & 81.8 & 46.7 & 0.001 \\
Going to a new clinic & 70.5 & 46.7 & 0.032 \\
Meeting a new doctor & 84.1 & 57.8 & 0.010 \\
\hline
\end{tabular}

Center, the patients and families and the adult $\mathrm{CF}$ Center. Allowing patients to interact with the adult team prior to transition and developing transition protocol that addresses CF-specific issues like infection control and fertility can lead to successful transition process from pediatric to adult CF care. ${ }^{9}$ There should be an evaluation of the outcome of the transition process on a regular basis. This would help in adjusting and modifying the process to meet patient needs. ${ }^{10}$

In a previous study, we evaluated our transition program that was established in $1980 .^{3}$ Forty patients attending the adult CF center completed a questionnaire. Patient opinions about the transition process and the pediatric and adult $\mathrm{CF}$ care centers were sought from these patients attending the adult $\mathrm{CF}$ center. All patients surveyed approved of having the visit with adult pulmonary physician in the pediatric clinic. Most thought that the transition program made the change from pediatric to adult care easier and they were comfortable leaving the pediatric clinic.

The major change that occurred as a result of our first study was to have an adult pulmonologist, not the adult pulmonary fellow, come to the pediatric clinic during the transition process. This change made the transition process easier for patients since they no longer needed to switch care providers twice. Also, an adult inpatient nurse coordinator was added in addition to the outpatient adult coordinator. A third change was to implement regular interactions between pediatric and adult teams and sharing of medical records.
In this study, we evaluated our transition process in an attempt to improve and modify it. Patients followed in the center fall into two categories, either transitioned from our pediatric program or referred to our center from other institutions. Because of the heterogeneity in the adult patient population, we conducted this study to help evaluate the transition process in our center.

Patients transferred to our adult center without a transition process were sicker with 33 patients of 47 (70\%) having moderate to severe disease. That is compared to 22 patients of $44(50 \%)$ that were formally transitioned to the adult program. In addition, the first group was on average, older at the time of transfer compared to the second group $(21.6 \pm 6.0$ vs. $19.1 \pm 2.1)$. The reason for the discrepancies could be due to the first group being lost to follow up for a period of time prior to transferring to our center. Other reasons could include leaving their pediatric center due to dissatisfaction, conflict, or lack of having an adult program. From this data, we can conclude that a younger age of transition (18-21 years) and healthier patients are a marker for successful transition.

Preparation for transition during the years preceding and the actual process of transition are very important to the success of the transition of care to adults. To complete the transition process, follow up appointments in the adult clinic are monitored closely by the pediatric program and the adult program as well, to ensure that patient care is not interrupted. In contrast, patients that were not transitioned were not monitored to ensure that

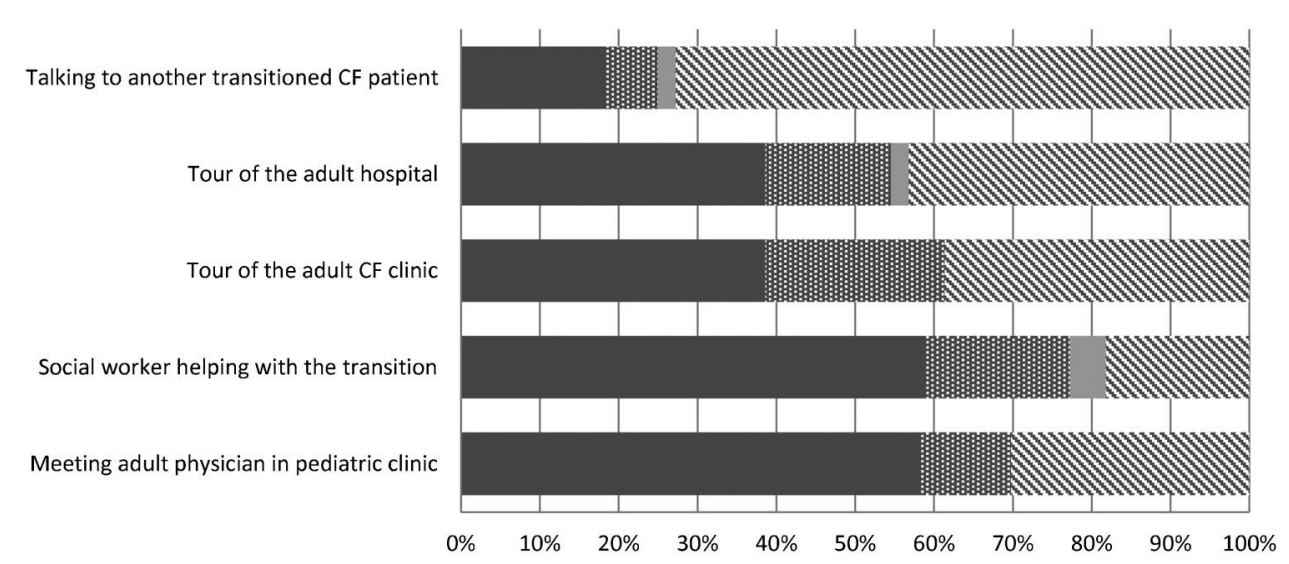

Fig. 2. Usefulness of $U$ of $M$ transition program experiences. $\square$, useful; ${ }^{4}$, somewhat useful; , not at all useful; $\mathbb{N}$, not experienced. 
they were seen in the adult clinic without interruption of care. Contacting our adult program depended on the non-transitioned patients leading to gaps in care. This can explain the difference in age and severity of disease between the two groups.

Tables 2 and 3 show that patients that participated in the transition program reported a higher level of satisfaction with care before transferring to the adult center, higher perceived health status both before and after the transition process. However, there was no statistical significance between independence or anxiety before and after the transition process. That suggests that the current transition program is insufficient in these two areas. These deficiencies will be focused on to improve our transition process in the future.

Analysis of the patients that went through our structured transition process (34 patients) versus patients that went through unstructured transition process (10 patients) indicated that there was a significant statistical difference between satisfaction with the pediatric program before and after the transition process $(P=0.001$ and 0.006 , respectively). This indicates that having a structured transition is crucial to patient satisfaction with their pediatric program. It would also lead to a successful transition to adult care.

Table 4 shows that patients who participated in the transition program were more likely to have talked to someone in the pediatric center, about leaving the pediatric clinic, leaving their pediatric physician, going to a new clinic, and meeting a new doctor $(P=0.001$, $0.001,0.032$, and 0.010 , respectively). This suggests that our pediatric center is more involved in transferring patient care to the adult center. Additionally, patients attending the pediatric center are more likely to receive guidance and support from their pediatric team throughout the transition process.

Even though all participants in the transition program went through the full transition process, which includes discussing all items in Table 4 (leaving the pediatric clinic, leaving their pediatric physician, going to a new clinic, and meeting a new doctor) with the social workers and other pediatric team members, some patients did not recall discussing these items with the team members $(25 \%, 19.2 \%, 29.5 \%$, and $15.9 \%$, respective1y). This could be due to the fact that the survey was obtained from transition program participants that transferred from pediatric to adult care on an average of 8.4 years prior. We believe that it was difficult for patients transitioned earlier to remember all items of the transition process especially since the transition time is usually a stressful time for them.

Participants in the $\mathrm{U}$ of $\mathrm{M}$ transition program reported that the most useful experience was meeting an adult physician in the pediatric clinic, followed closely by having a social worker help in the transition process. Tours of the adult CF clinic and the adult hospital are less likely to have occurred. Figure 2 suggests that these tours have been useful for the participants that experienced them. One change that will be implemented in our center is having the tour of the inpatient and outpatient facilities as integral part of the transition process. Talking to another CF patient who already transitioned is not a routine part of our transition program because of infection control measures. However, most participants that did experience this found it to be at least somewhat useful. Currently, with the help of the parent advisory board and input from patients, we created an electronic communication through email. In addition, a web based communication that will enable patients to communicate, especially around transition time, without worrying about infection control measures will be investigated. Our findings suggest that CF centers should continue to formalize their transition process to optimize patient health, continuity of care, and hopefully adherence to their treatment plan. It also can lead to decrease lost to follow up and increase satisfaction with the pediatric center and its staff. In the future, the transition experience in CF can serve as a model for other chronic illnesses.

A limitation to the study is that it is a retrospective, self-report study. A prospective transition evaluation would be required to provide more data on the efficacy of the process.

\section{ACKNOWLEDGMENTS}

The authors would like to thank the patients for participating in the study and Dr. Richard Simon and the adult team for their feedback and assistance in conducting the study.

\section{REFERENCES}

1. Blum RW. Transition to adult health care: setting the stage. J Adolesc Health 1995; 17:3-5.

2. American Academy of Pediatrics. American Academy of Family Physicians, American College of Physicians, Transitions Clinical Report Authoring Group. Clinical report-supporting the health care transition from adolescence to adulthood in the medical home. Pediatrics 2011;128:182-200.

3. Nasr SZ, Campbell C, Howatt W. Transition program from pediatric to adult care for cystic fibrosis patients. J Adolesc Health 1992;13:682-685.

4. Lugasi T, Achille M, Stevenson M. Patients' perspective on factors that facilitate transition from child-centered to adultcentered health care: a theory integrated metasummary of quantitative and qualitative studies. J Adolesc Health 2011;48: $429-440$.

5. Kennedy A, Sloman F, Douglass JA, Sawyer SM. Young people with chronic illness: the approach to transition. Intern Med J 2007;37:555-560.

6. Tuchman LK, Slap GB, Britto MT. Transition to adult care: experiences and expectations of adolescents with a chronic illness. Child Care Health Dev 2008;34:557-563. 
7. Nakhla M, Daneman D, To T, Paradis G, Guttman A. Transition to adult care for youths with diabetes mellitus: findings from a universal health care system. Pediatrics 2009;124:e1134-e1141.

8. Flume PA, Anderson DL, Hardy KK, Gray S. Transition programs in cystic fibrosis centers: perceptions of pediatric and adult program directors. Pediatr Pulmonol 2001;31:443-450.

9. Boyle MP, Farukhi Z, Nosky ML. Strategies for improving transition to adult cystic fibrosis care, based on patient and parent views. Pediatr Pulmonol 2001;32:428-436.

10. Madge S, Bryon M. A model for transition from pediatric to adult care in cystic fibrosis. J Pediatr Nurs 2002;17:283-288.

11. Anderson DL, Flume PA, Hardy KK, Gray S. Transition programs in cystic fibrosis centers: perceptions of patients. Pediatr Pulmonol 2002;33:327-331.

12. Flume PA. Smoothing the transition from pediatric to adult care: lessons learned. Curr Opin Pulm Med 2009;15:611-614.
13. Mclaughlin SE, Diener-West M, Indurkhya A, Rubin H, Hechmann R, Boyle MP. Improving transition from pediatric to adult cystic fibrosis care: lessons from a national survey of current practices. Pediatrics 2008;121:e1160-e1166.

14. Crowley R, Wolfe I, Lock K, McKee M. Improving the transition between paediatric and adult healthcare: a systematic review. Arch Dis Child 2011;96:548-553.

15. Cystic Fibrosis Foundation. Patient Registry: Annual Data Report 2010.

16. Craig SL, Phty B, Towns S, Bibby H. Moving on from paediatric to adult health care: an initial evaluation of a transition program for young people with cystic fibrosis. Int $\mathbf{J}$ Adolesc Med Health 2007; 19:333-343.

17. Dejong G, Paslbo SE, Beatty PW, Jones GC, Krolls T, Neri MT. The organization and financing of health services for persons with disabilities. Milbank Q 2002;80:261-301. 